\title{
Development of assisted reproductive
} technology services in Thailand between 2008 and 2014 before the new law: Results generated from the National ART Registry, Royal Thai College of Obstetricians and Gynecologists

\author{
Charoenchai Chiamchanya', Kamthorn Pruksananonda ${ }^{2, *}$
}

\begin{abstract}
Background: While the assisted reproductive technology (ART) relieves the burden of infertility in many couples, it presents significant public health challenges due to the substantial risk for multiple birth delivery and preterm birth, which are associated with poor maternal and fetal health outcomes. For this reason, it is important to monitor the development and effectiveness of ART services in Thailand.

Objective: To analyze the trends of ART services in Thailand between 2008 and 2014.

Methods: ART clinics in Thailand are required to submit data to the Royal Thai College of Obstetricians and Gynecologists via the National Reporting System. The data from 2008 to 2014 were collected and analyzed.

Results: The number of ART centers was increased from 35 to 47 . The total fresh ART cycles were also increased from 3,723 to 6,516. The percentage values of intracytoplasmic sperm injection (ICSI), in vitro fertilization, gamete intrafallopian transfer, and zygote intrafallopian transfer cycles were changed from 77.87 to $95.59,21.43$ to $4.31,0.21$ to 0.09 , and 0.45 to 0.05 , respectively. The clinical pregnancy rates were $28.79-33.19,22.84-51.34,14.29-42.86$, and $0.00-26.67$, respectively. The clinical pregnancy rates in fresh vs. frozen-thawed cycles were $31.01-36.33$ vs. $31.54-37.34(P<0.05)$. The clinical pregnancy rates in female age $<35$ vs. $35-39$ vs. $\geq 40$ years were $36.97-40.70$ vs. $32.74-33.42$ vs. $21.08-$ 31.34 , respectively $(P<0.001)$, and the percentage values of multifetal pregnancy rate were 18.75 vs. 13.30 and 13.69 , respectively $(P<0.001)$. There were increasing preimplantation genetic screening (PGS) cycles, with the percentage of the clinical pregnancy rate $(25.90-42.63, P<0.05)$. The clinical pregnancy rates in medium-sized ART centers $(100-300$ cycles per year) vs. in small and large centers were $30.79-41.14$ vs. $28.01-34.04$ and $8.70-40.35$, respectively $(P<0.001)$. Trends of increasing percentage of ART birth rate to total birth rate ratio were $0.24-0.34(P<0.05)$.

Conclusions: There were higher clinical pregnancy rates in frozen-thawed cycles. Higher multifetal pregnancy rate and clinical pregnancy rate were also found in younger females. There were increasing uses of ICSI and PGS. A trend toward increasing ART birth to total birth ratio was observed.
\end{abstract}

Keywords: assisted reproductive technology; egg donor; infertility; IVF; surrogacy

\footnotetext{
*Correspondence to: Kamthorn Pruksananonda, Department of Obstetrics and Gynecology, Faculty of Medicine, Chulalongkorn University, Bangkok 10330, Thailand, e-mail: Kamthorn.P@chula.ac.th; pkamthorn@yahoo.com

'Department of Obstetrics and Gynecology, Faculty of Medicine, Thammasat University, Pathumthani 12120, Thailand

${ }^{2}$ Department of Obstetrics and Gynecology, Faculty of Medicine, Chulalongkorn University, Bangkok 10330, Thailand
}

¿ Open Access. ๑ 2019 Chiamchanya and Pruksananonda, published by Sciendo. (c) BY-NC-ND This work is licensed under the Creative Commons Attribution NonCommercial-NoDerivatives 4.0 License. 
Infertility is a worldwide health problem that affects 48.5 million couples [1]. It is commonly found in a population with delayed marriage or those trying to conceive at an older age, which decreases fecundity. This problem is more pronounced in developed countries whose population growth has declined but the burden of infertility and its social consequences are predominantly found in developing countries. In response to the increasing infertility problem, rapid progress in research, novel technology, and treatment and care for the infertile were developed [2]. Assisted reproductive technology (ART) is the routine common and successful treatment. Almost, all of the published research articles in ART were from developed countries. This technology was then passed onto the developing countries with or without modifications. The ART registry data are also mostly from developed countries [3]. A number of the new treatments have been integrated into routine practices yielding different outcomes in various regions, which has raised the question of copy and adoption of policy or recommendation of global ART practice. This may have a positive or negative consequence for Japan. Its live birth rate in fresh cycle with minimal stimulation protocol and implementation of elective single embryo transfer (SET) dropped to $5 \%$. The rate for multifetal pregnancy in 2012 was $<4 \%$. This may triple the number of annual ART cycles [3]. The ART registry data from different regions and various socioeconomic countries will be useful in adopting and adjusting the policy to develop global ART practices. The ART registry data reports or researches from developing countries are scanty. Most of them are from large countries such as India, Iran, Brazil, and China [2, 3]. Thailand, with 67 million people, is a developing country in Southeast Asia, and it is one of the earliest countries in Asia to introduce in vitro fertilization (IVF) techniques. The population growth has dramatically dropped to $0.4 \%$ and its total fertility rate in 2015 was $1.6 \%$. Hence, the health policy was shifted from fertility control to fertility treatment. The first IVF baby was born in 1987 by Virutamasen, at the Faculty of Medicine, Chulalongkorn University. Thailand's "dek lord kaew" (glass tube child) was described as the 2,999th baby to be born through IVF worldwide (the three nines making this an especially auspicious number for Thais), which was noted a year before China and nearly a decade after the birth of Louise Brown in 1978 in Britain [4]. Since that time, newer technology such as intracytoplasmic sperm injection (ICSI) has been developed very fast successfully [5]. It is obvious that there is a need for more ART units and services. However, the ART registry data that represent the fertility situation and fertility healthcare services are very limited. Only one record by Vutyavanich done in 2011 was found [6]. In Thailand, the pregnancy rate per transfer in fresh cycle with number of embryo transfer between 2.9 and
4.1 was between 30.7 and $38.6 \%$. There are no data about live birth rate. On the other hand, the live birth rate with elective SET was $28-29 \%$ in the USA, $17-25 \%$ in Europe, $24-25 \%$ in the UK, $19-20 \%$ in Australia, and 9-15\% in Japan [3]. In 2009, there were changes to the ART policy in Europe. The legal marriage or a stable union is often a prerequisite for ART treatment. Some types of ART services such as surrogacy are strictly prohibited in many European countries. However, this can be easily circumvented by crossing national boundaries for ART treatments [7]. India and Thailand are well-known destinations for reproductive tourisms and they have become a hub for ART services [8]. In 2012, India has a new medical policy restricting surrogacy, so surrogacy at present moves into Thailand $[9,10]$. ART treatments in Thailand are of high quality and provide exceptional services. However, surrogacy cross-border-assisted reproductive care raises many ethical and social issues [11]. One familiar example of this happened in 2013 in which one of the twins, well known as baby Gammy, with mental retardation was left behind by the surrogacy clients $[8,12]$. As a result of this, Thailand has launched a new ART law to control and regulate ART services in preventing such incidences from happening again. It also revised the new data registry system. This study will summarize and analyze trends of ART and its National Data Registry System in Thailand between 2008 and 2014, before the ART law.

\section{Materials and methods}

This is a retrospective study. ART data from all registered ART centers submitted to the Royal Thai College of Obstetricians and Gynecologists (RTCOG) between 2008 and 2014 were reviewed. This study was approved by the Reproductive Medicine Subcommittee and RTCOG in April 2019. The annual report form was designed by RTCOG. The report forms were divided into three subgroups according to the females' age $<35,35-39$, and $\geq 40$ years. The report also collected the following data: the number of both fresh and frozen-thawed cycles, type of ART, number of oocyte retrieval, number of fertilized oocyte, number of fresh embryo transfer, number of frozen embryo, number of thawed embryo, number of frozen-thawed embryo transfer, the result of treatment, number of clinical pregnancy, number of pregnancy loss, number of ectopic pregnancy, the outcome of pregnancy, and number of preimplantation genetic screening (PGS) cycle and its results. Clinical pregnancy was defined as the presence of a gestational sac with or without fetal heartbeat analyzed using ultrasonography. Pregnancy loss was defined as miscarriage or pregnancy termination before 20 weeks of gestation. The live birth rate was defined as a delivery that resulted in at least 
one living infant. The outcome of pregnancy such as singleton, twins, triplets, quadruplets, or more was defined as the delivery of $\geq 1$ infant, either alive or stillbirth. In order to overcome the ongoing pregnancy result, the schedule of the annual ART data report sent to the RTCOG was postponed from the previous March to October. In October of every year, the RTCOG sends a request letter and ART data report form to all registered ART centers. Most of the data at ART centers are in hard copy. Some centers use simple computer programs such as Microsoft Excel to record the data. Few centers could assess to dedicated ART data collection programs. Few centers did not record any data, and therefore they were unable to report back to the RTCOG. At the end of each year, complete annual ART data between January and December had to be validated by the director of each center before it is sent to the RTCOG. The annual ART registry data were manually checked and the incomplete data of each ART center were notified by RTCOG for clarification.

However, on-site monitoring of the data was not performed. Therefore, it cannot be said with $100 \%$ certainty that the numbers transcribed onto the report form match the source documents. All ART centers that want to continue to provide services must complete the annual ART report form. The data from all ART centers were aggregated for data analysis. Data from individual ART centers were treated as classified and were kept anonymous. Only the summarized data were made available to the public. The primary and secondary outcomes assessments were the number of ART center, type of ART, the number of both fresh and frozen-thawed ART cycles, the clinical pregnancy rate, the outcome of pregnancy, the results in each female age subgroup, the number of PGS cycle and its results, the size of ART center and its success rate, and the ART to total birth rate ratio. The descriptive data were presented as percentages. The comparative study was analyzed by the chi-squared test with significant level at 0.05 . The trends were exhibited by graphics.

\section{Results}

The number of ART centers in Thailand increased from 35 in 2008 to 47 in 2014 and also the total fresh ART cycles increased from 3,723 in 2008 to 6,516 in 2014. The proportion of each type of ART treatment showed that ICSI increased from $2,907(77.87 \%)$ in 2008 to $6,229(95.59 \%)$ in 2014 , whereas IVF, gamete intrafallopian transfer (GIFT), and zygote intrafallopian transfer (ZIFT) decreased from 799 (21.43\%), 8 $(0.21 \%)$, and $17(0.45 \%)$ in 2008 to $281(4.31 \%), 6(0.09 \%)$, and $3(0.05 \%)$ in 2014 , respectively. The total frozen-thawed embryo transfer (FET) cycles also increased from 1,712 in 2008 to 6,240 in 2014 (Figures 1 and 2).
From 2008 to 2014, the clinical pregnancy rates per transfer with ICSI (surgical sperm retrieval), ICSI (ejaculate sperm), IVF, ZIFT, and GIFT were between 14.48 and $34.00 \%, 28.79$ and $33.19 \%, 22.84$ and $51.34 \%, 0.00$ and $26.67 \%$, and 14.29 and $42.86 \%$, respectively (Figure 3).

In fresh cycle, the clinical pregnancy rates per transfer in $2008-2014$ were $36.33 \%, 32.88 \%, 31.28 \%, 31.01 \%, 32.15 \%$, $32.27 \%$, and $32.84 \%$, respectively. The live birth rates in 2008, 2009, 2010, 2011, 2012, 2013, and 2014 were $27.63 \%$, $23.88 \%, 26.43 \%, 25.04 \%, 29.80 \%, 23.13 \%$, and $25.60 \%$, respectively.

The number of embryos per transfer in 2008-2014 were $2.41,2.80,2.49,2.25,2.33,2.21$, and 2.15 , respectively. Between 2008 and 2014, the percentage values of singleton, twins, triplets, quadruplets, or more in fresh cycle were $67.09-86.55,11.75-29.30,0.49-15.95$, and $0.00-11.82$, respectively. In the frozen-thawed cycle, the clinical pregnancy

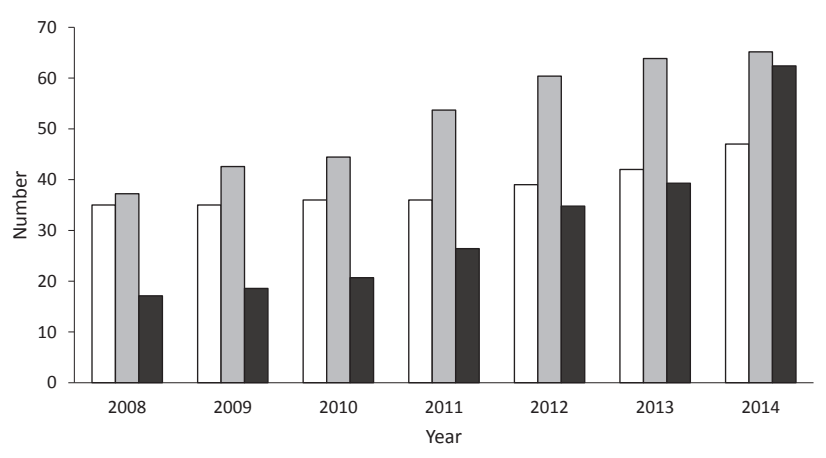

Figure 1. Trends of the number of ART centers (white bars) and total ART cycles (initiated cycles; light-gray bars and FET cycles; black bars) in Thailand (unit in hundred) between 2008 and 2014.

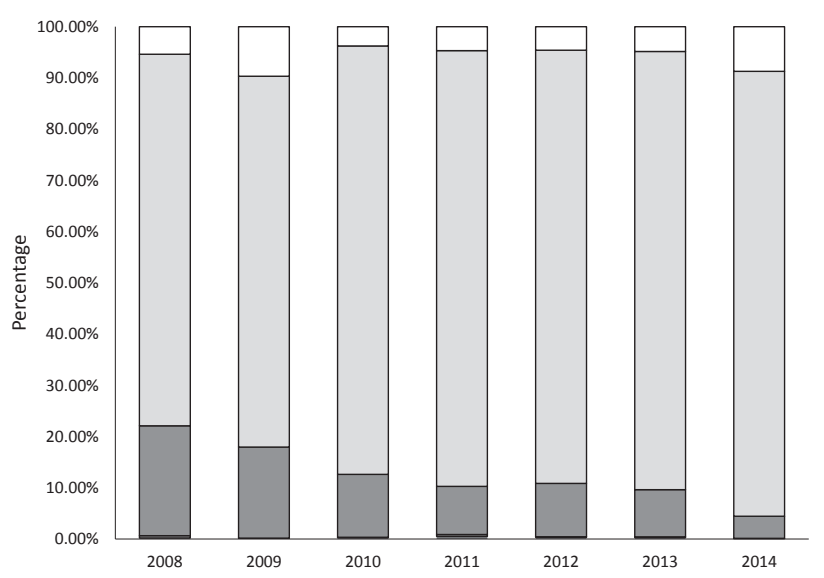

Figure 2. The proportion of ART techniques in Thailand between 2008 and 2014. The bars represent the ART techniques; ICSI surgically retrieved sperm (white bars), ICSI ejaculated sperm (light-gray bars), IVF (medium-gray bars), ZIFT (dark-gray bars), GIFT (black bars). 


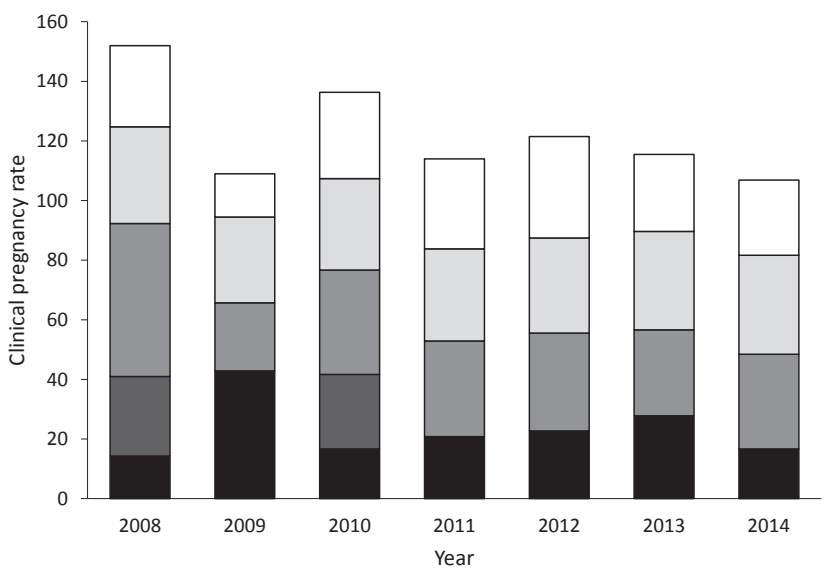

Figure 3. The clinical pregnancy rate per transfer of each ART techniques in Thailand between 2008 and 2014. The bars represent the ART techniques; ICSI surgically retrieved sperm (white bars), ICSI ejaculated sperm (light-gray bars), IVF (medium-gray bars), ZIFT (dark-gray bars), GIFT (black bars).

rates per transfer in $2008-2014$ were $34.27 \%, 34.37 \%$, $31.54 \%, 37.34 \%, 35.00 \%, 36.81 \%$, and $37.11 \%$, respectively. The live birth rates in $2008-2014$ were $33.68 \%, 25.55 \%$, $30.64 \%, 28.69 \%, 29.18 \%, 22.77 \%$, and $28.27 \%$, respectively. The number of embryos per transfer in 2008-2014 were 2.54, $2.37,2.30,2.45,2.24,2.12$, and 1.93 , respectively.

From 2008 to 2014, the percentage values of singleton, twins, triplets, quadruplets, or more in frozen-thawed cycle were $53.57-81.03,15.68-25.59,0.70-3.72$, and $0.00-0.21$, respectively. When fresh cycle was compared with the frozenthawed cycle, the clinical pregnancy rates per transfer from 2008 to 2014 were higher for the frozen-thawed cycle except in 2008 and 2010 (36.36\%*, 32.88\%*, 31.28\%, 31.01\%**, $32.15 \% *$, $32.27 \% * *, 32.84 \% * *$ vs. $34.27 \% *$, $34.37 \% *$, $31.54 \%, 37.34 \%^{* *}, 35.00 \% *$, 36.81\%**, 37.11\%**, respectively; ${ }^{*} P<0.05,{ }^{* *} P<0.01$ ) (Figure 4).

\section{Fresh cycle between each female age group}

The clinical pregnancy rates per transfer for females aged $<35$, $35-39$, and $\geq 40$ years decreased $(36.97 \%, 32.74 \%$, and $21.08 \%$ respectively; $P<0.001)$. The live birth rates for females aged $<35,35-39$ and $\geq 40$ years also decreased $(31.34 \%, 25.37 \%$, and $14.61 \%$, respectively; $P<0.001)$. The percentage values of singleton, twins, triplets, quadruplets, or more were different between the age groups $\left({ }^{*} P<0.001\right.$ and $\left.{ }^{* *} P<0.05\right)$. The percentage values of singleton, twins, triplets, quadruplets, or more, and no data available for females aged $<35$ years, were $59.82 \% *, 16.40 \% * *, 2.14 \% * *, 0.21 \%$, and $21.42 \%$, respectively. The percentage values of singleton, twins, triplets,

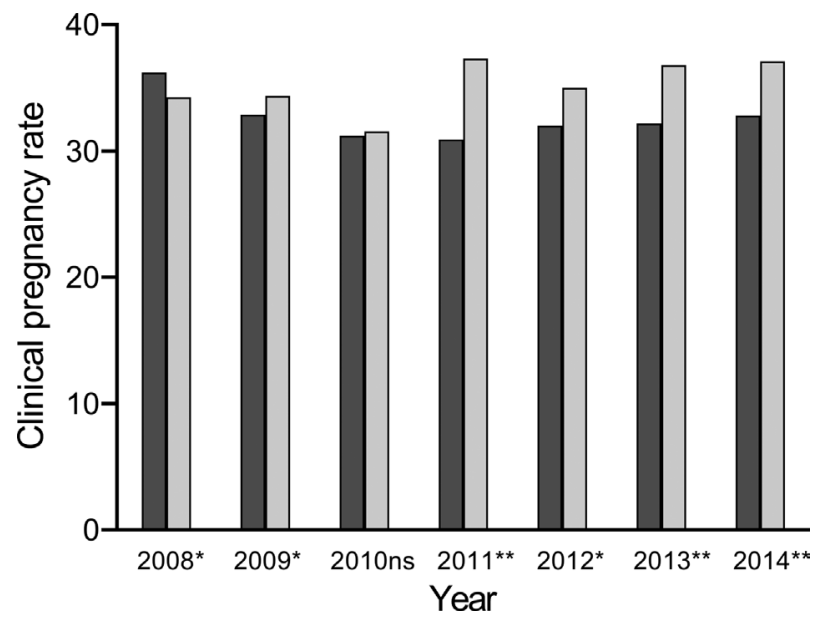

Figure 4. Comparison of the clinical pregnancy rate per transfer between fresh (dark-gray bars) and frozen-thawed (light-gray bars) cycles in Thailand between 2008 and 2014. ${ }^{*} P<0.05 ;{ }^{* *} P<0.01 ; \mathrm{ns}=P>0.05$.

quadruplets, or more, and no data available for females aged $35-39$ years, were $64.20 \% *, 11.87 \%, 1.43 \%, 0.00 \%$, and $22.49 \%$, respectively. The percentage values of singleton, twins, triplets, quadruplets, or more, and no data available for females aged $\geq 40$ years, were $71.54 \% * 12.60 \%, 1.09 \%$, $0.00 \%$, and $14.77 \%$, respectively. The clinical pregnancy loss rate and ectopic pregnancy rate were different between the age groups $(* P<0.001$ and $* * P=0.009)$. The clinical pregnancy loss rate and ectopic pregnancy rate for females aged $<35$ years were $13.06 \% *$ and $2.17 \% * *$, respectively.

The clinical pregnancy loss rate and ectopic pregnancy rate for females aged 35-39 years were 20.76\%* and 1.75\%*, respectively. The clinical pregnancy loss rate and ectopic pregnancy rate for females aged $\geq 40$ years were $28.23 \% *$ and $2.48 \% *$, respectively.

\section{Frozen-thawed cycle between each female age group}

The clinical pregnancy rates per transfer for females aged $<35,35-39$, and $\geq 40$ years decreased $(40.70 \%, 33.42 \%$, and $31.34 \%$, respectively; $P<0.001$ ). The live birth rates for females aged $<35,35-39$, and $\geq 40$ years also decreased (35.54\%, $27.77 \%$, and $23.83 \%$, respectively; $P<0.001)$. The percentage values of singleton, twins, triplets, quadruplets, or more were different between the age groups $(* P<0.001)$. The percentage values of singleton, twins, triplets, quadruplets, or more, and no data available for females aged $<35$ years, were $57.20 \% *, 18.18 \%, 1.39 \%, 0.00 \%$, and $23.23 \%$, respectively. The percentage values of singleton, twins, triplets, quadruplets, or more, and no data available for females aged 35-39 years, were $61.92 \% *, 17.61 \%, 0.95 \%, 0.00 \%$, and $19.52 \%$, respectively. The percentage values of singleton, twins, triplets, 
quadruplets, or more, and no data available for females aged $\geq 40$ years, were $65.96 \% *, 16.15 \%, 0.58 \%, 0.00 \%$, and $17.31 \%$, respectively. The clinical pregnancy loss rate and the ectopic pregnancy rate were different between the age groups $(P<0.001)$. The clinical pregnancy loss rate and the ectopic pregnancy rate for females aged $<35$ years were $12.02 \%$ and $0.66 \%$, respectively. The clinical pregnancy loss rate and the ectopic pregnancy rate for females aged 35-39 years were $14.95 \%$ and $1.95 \%$, respectively. The clinical pregnancy loss rate and the ectopic pregnancy rate for females aged $\geq 40$ years were $22.81 \%$ and $0.17 \%$, respectively. The clinical pregnancy rates per transfer for fresh and FET cycles between the age groups were different (Figure 5).

The number of the PGS cycles in 2008-2014 were 557, $912,794,1,419,1,511,1,804,2,811$ of which $14.96 \%, 21.41 \%$, $17.87 \%, 26.43 \%, 25.02 \%, 28.20 \%, 43.14 \%$ were of total ART cycles. The clinical pregnancy rates per initiated cycle and per retrieval cycle with PGS using fluorescent in situ hybridization (FISH) technique in 2008-2014 decreased (between 9.53 and $25.20 \%$ and 9.78 and $26.95 \%$, respectively; $P<0.05$ ). The clinical pregnancy rates per transfer cycle with PGS using FISH technique increased in 2008-2013 and decreased in 2014 (between 25.90 and 42.63\%, $P<0.05$ ) (Figure 6).

From 2008 to 2014, except for 2009, 2012, and 2013, the clinical pregnancy rates per transfer were higher in mediumsized centers (100-400 cycles per year) compared with
Pregnancy rate per transfer

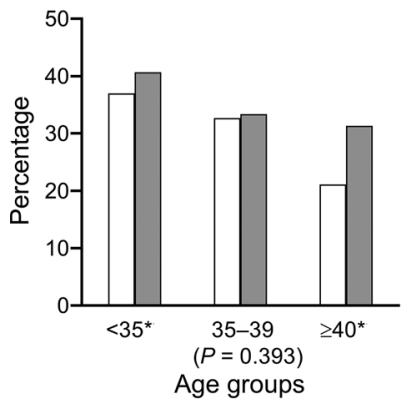

Live birth rate

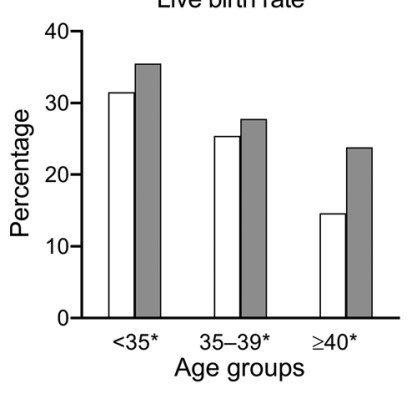

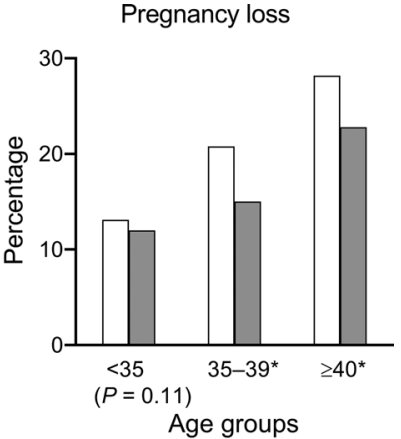

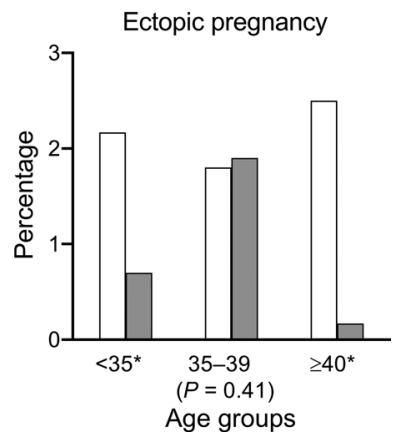

Figure 5. Comparison of the clinical pregnancy rate per transfer and the pregnancy results among different female partner age groups between fresh cycle (white bars) and frozen-thawed cycle (gray bars) in Thailand between 2008 and 2014. ${ }^{*} P<0.01$. small-sized centers $(<100$ cycles per year). From 2008 to 2014, except for 2009, 2013, and 2014, the clinical pregnancy rates per transfer were higher in medium-sized centers (100400 cycles per year) compared with the large-sized centers ( $>400$ cycles per year) $(P<0.05, \mathrm{OR}=1.06-1.66 ; 95 \% \mathrm{CI})$ (Table 1). The percentage of the ratio of ART birth rate to total birth rate increased from $0.24 \%$ in 2008 to $0.34 \%$ in 2014 $(P<0.05)$ (Figure 7).

\section{Discussion}

From 2008 to 2014, both fresh and FET ART cycles increased, most of them were ICSI. On the other hand, IVF, GIFT, and ZIFT decreased. However, the clinical pregnancy rates per transfer with ICSI, IVF, GIFT, and ZIFT did not increase. The number of ART centers increased from 35 in 2008 to 47 in 2014. Medium-sized centers with 100-400 cycles per year had more success rates for clinical pregnancy rates per transfer. However, this rate decreased momentarily in 2013 and improved in 2014. The success rate for small centers $(<100$ cycles per year) peaked in 2009 and 2013. As for large centers (>400 cycles), the success rates dropped in 2011 and 2012 and peaked in 2014. This finding is different from the data between 2001 and 2007 which showed large centers ( $>400$ cycles per year) having sustained their high success rates.

From 2008 to 2014, there is an increasing trend for ART to total birth rate ratio which was between 0.24 and $0.34 \%$. This was 5-10 times lower compared with developed countries. It was $1.6 \%$ in USA [13], 3-5\% in Europe [14], and 3\% in Australia [15]. The clinical pregnancy rates per transfer in FET

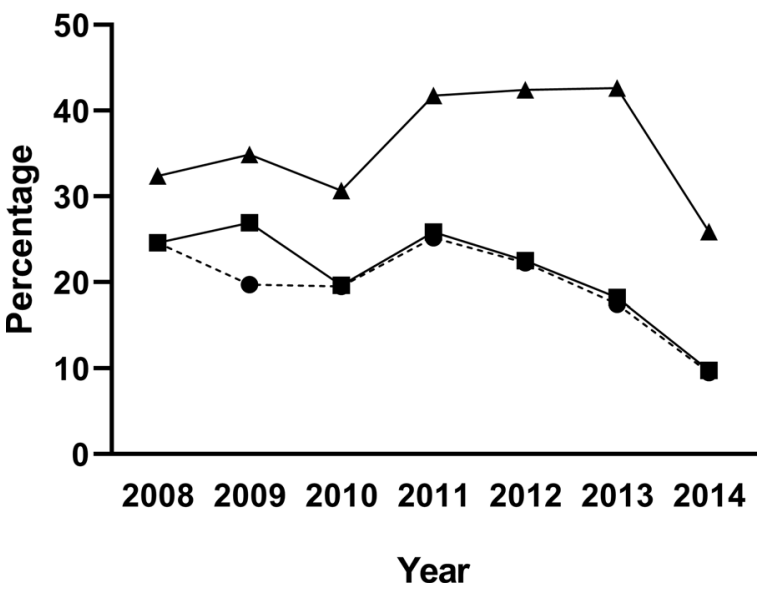

Figure 6. Comparison trend of the clinical pregnancy rates with PGS cycles in Thailand between 2008 and 2014. The lines show pregnancies/ initiated cycles (circles, dashed lines), pregnancies/retrieved cycles (squares, unbroken lines), and pregnancies/transfer cycles (triangles, unbroken lines). 
Table 1. Comparison of the clinical pregnancy rate per transfer between the size of ART centers in Thailand in 2008-2014

\begin{tabular}{|c|c|c|c|c|c|c|}
\hline Year & Cycles & No. of centers & Total cycles & Pregnancies/transfer cycle (\%) & $P^{*}$ & OR $(95 \% \mathrm{Cl})$ \\
\hline \multirow[t]{3}{*}{2008} & $<100$ & 22 & 797 & 28.01 & - & 1 \\
\hline & $100-400$ & 11 & 1,953 & 35.31 & $<0.01$ & $1.41(1.18,1.69)$ \\
\hline & $>400$ & 2 & 872 & 28.01 & 0.99 & $1.0(0.81,1.24)$ \\
\hline \multirow[t]{3}{*}{2009} & $<100$ & 16 & 454 & 34.04 & - & 1 \\
\hline & $100-400$ & 16 & 2,962 & 35.66 & 0.53 & $1.06(0.87,1.32)$ \\
\hline & $>400$ & 5 & 3,065 & 34.57 & 0.85 & $1.02(0.83,1.25)$ \\
\hline \multirow[t]{3}{*}{2010} & $<100$ & 23 & 1,139 & 32.89 & - & 1 \\
\hline & $100-400$ & 11 & 2,370 & 36.67 & 0.03 & $1.18(1.02,1.37)$ \\
\hline & $>400$ & 2 & 978 & 31.96 & 0.66 & $0.96(0.80,1.15)$ \\
\hline \multirow[t]{3}{*}{2011} & $<100$ & 22 & 1,045 & 30.86 & - & 1 \\
\hline & $100-400$ & 12 & 2,384 & 34.48 & 0.04 & $1.19(1.01,1.39)$ \\
\hline & $>400$ & 3 & 1,726 & 17.83 & $<0.01$ & $0.48(0.4,0.58)$ \\
\hline \multirow[t]{3}{*}{2012} & $<100$ & 19 & 736 & 31.52 & - & 1 \\
\hline & $100-400$ & 17 & 3,438 & 34.01 & 0.2 & $1.12(0.94,1.33)$ \\
\hline & $>400$ & 3 & 1,707 & 8.7 & $<0.01$ & $0.21(0.16,0.26)$ \\
\hline \multirow[t]{3}{*}{2013} & $<100$ & 18 & 618 & 33.32 & - & 1 \\
\hline & $100-400$ & 20 & 3,546 & 30.79 & $<0.01$ & $1.66(1.37,1.96)$ \\
\hline & $>400$ & 4 & 2,260 & 33.01 & 0.88 & $0.99(0.81,1.19)$ \\
\hline \multirow[t]{3}{*}{2014} & $<100$ & 27 & 1,294 & 31.04 & - & 1 \\
\hline & $100-400$ & 16 & 2,900 & 41.14 & $<0.01$ & $1.55(1.34,1.78)$ \\
\hline & $>400$ & 4 & 2,142 & 40.35 & $<0.01$ & $1.50(1.29,1.74)$ \\
\hline
\end{tabular}

*From chi-squared test compare with cycle $<100$.

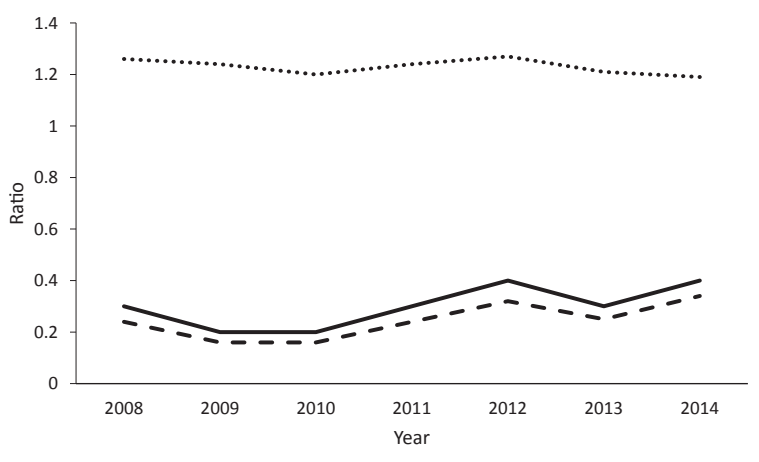

Figure 7. Trends of the percentage of the ratio of ART birth rate to natural birth rate in Thailand between 2008 and 2014. The lines show birth rate from ART/1000 (unbroken lines), national birth rate/100 (dotted lines), and birth rate from ART/natural birth rate (\%) (dashed lines).

cycles were higher than fresh transfer cycles except in 2008 and 2010. This is similar to the USA in 2013 when FET with nondonor had a higher pregnancy rate compared with the fresh transfer cycle. In contrast, in the UK, the live birth rate per transfer in fresh transfer cycle was higher than in FET cycle in 2013. Likewise, in Europe in 2011, the pregnancy rate per aspiration in fresh transfer cycle was higher than the pregnancy rate per thawing in FET cycle [16]. In 2006, the ICMART data for pregnancy rate per aspiration in fresh transfer cycle was higher than the pregnancy rate for FET cycle [17].

The clinical pregnancy rate and the live birth rate per transfer for both fresh transfer and FET cycles were highest among females aged $<35$ years followed by females aged 35-39 years. The clinical pregnancy loss rates for both fresh transfer and FET cycles were highest in females aged $\geq 40$ years followed by females aged 35-39 years. However, there were more singleton pregnancy rates in females aged $\geq 40$ years for both fresh transfer and FET cycles. There were more multifetal pregnancy rates in females aged $<35$ years, especially for triplets with fresh transfer cycles. There were no prematurity and newborn anomaly data available. There is an increasing number of PGS cycles from 2008 to 2014 using the FISH technique. The clinical pregnancy rate per transfer increased except in 2014. However, the clinical pregnancy rate per initiated cycles and retrieval cycles decreased and were lower than those of the non-PGS cycles. This is more or less similar to the current data using this technique [18]. This study found that the trends of ART methods in Thailand 
shifted from the more invasive methods such as GIFT and ZIFT to the less invasive and higher quality controlled laboratory methods like IVF and ICSI, as seen in the USA [19] and European countries [16].

However, there were higher ratios of ICSI/IVF cycles in Thailand (95.59\%:4.31\%) when compared with the USA (69\%:31\%), Europe (68\%:32\%) [16], Australia and New Zealand (63\%:37\%) [15], and ICMART data (66\%:34\%) [17]. There was also a higher rate of PGS cycles in Thailand (14.96$43.14 \%$ ) compared with those of $6 \%$ of total ART cycles in the USA in 2013, 1.12\% of total ART cycles in Europe in 2011 [16], and $4.95 \%$ of total ART cycles in Australia and New Zealand in 2014 [15].

The higher ICSI/IVF cycle ratio and PGS/non-PGS cycle ratio may represent the liberal use of higher technologies in ART in Thailand; however, the clinical pregnancy rates with these sophisticated technologies were not higher than those of nonuse. The result of ICSI is similar to that in the USA [20]. The FET cycles with higher clinical pregnancy rates per transfer may result in changing the service strategy to segmentation ART program in Thailand as the trend of FET cycles increased from $31.50 \%$ in 2008 to $48.92 \%$ in 2014 of total ART cycles. This finding is similar to that of Australia and New Zealand (47.1\% of total ART cycles in 2014) [15] compared with $24.5 \%$ in the USA in 2013 [19], $21.26 \%$ in Europe in 2011 [21], 23and 27.4\% in ICMART report in 2006 [17].

The lower clinical pregnancy rate per transfer in females aged $\geq 40$ years and higher multifetal pregnancy rate in females aged $<35$ years are still challenging in Thailand and worldwide [15, 16, 22]. In 2014, the number of embryos per transfer in both fresh and FET cycles is around 2.15 and 1.93, respectively. Maybe it is time to move SET to females aged $<35$ years as done in developed countries.

The number of ART centers and ART cycles in Thailand increased between 2008 and 2014. Trends of the success rate did not increase during that period but the clinical pregnancy rate per transfer was better than the data reported between 2001 and 2007 [6]. The reason for this may be due to the higher demand for ART services, so the quality improved even though the success rate was not as high as in the USA (45.4\%) in 2013 [19].

This study showed that ART service in Thailand is growing. With that increase, there are many other ethical and social aspects in ART services in Thailand that need to be considered such as the cross-border commercial surrogacy as seen in the case of baby Grammy, homosexual male couple, a Japanese man with many surrogate mothers and newborns, Taiwanese couples with Vietnamese surrogate mothers [12]. Due to this, there is an urgent launch of the new ART law. In 2015, the new ART law was implemented in Thailand. This new law now requires the marriage certificate of the infertile couple, and insurance that the right and the social welfare of the child born by ART are in place. ART services are now strictly regulated. The PGS cannot be done for social sex selection, and the third-party reproductive care services especially in either commercial or cross-border surrogacy and in commercial oocyte donation are not allowed. Surrogacy and oocyte donation are not allowed cross border. Previously, Thailand had no law regulating or addressing the issue of surrogacy. The legal authorities usually applied a provision in the Criminal Code or the Civil and Commercial Code, which states that anyone who enslaves another person or causes a person to be in a position similar to that of a slave; transports them into or out of the country; or buys, sells, disposes, accepts, or restrains a person may be subject to imprisonment for up to 7 years and fined up to 14,000 Baht (around US\$400). The woman giving birth to a child is that child's legal mother, and the only way for another woman to be recognized as the mother is through adoption. However, on February 19, 2015, the National Legislative Assembly of Thailand enacted the Protection for Children Born Through Assisted Reproductive Technologies Act (Surrogacy Act), which was enforced 90 days after it was published in the Royal Thai Government Gazette on May 1, 2015. This act significantly protects children born through ART and sets the legal procedures that the spouses must follow in order to have such legal children. It also prohibits sex selection. The main purposes of the new Surrogacy Act are as follows: (1) to specify the parents' legal status and protect the rights of the children; (2) to control and specify the rights and duties of related parties during and after surrogacy; (3) to control and set boundaries on the proper use of enhanced ARTs, especially for achieving pregnancy; and (4) to prohibit surrogacy involving a business or profit-making enterprise through agencies. This Act will end commercial surrogacy in Thailand. After the new ART law was implemented, the ART service system in Thailand was reformed and several new ART centers were established. In collaboration with the Ministry of Public Health, the Ministry of Social Development and Human Security, the Ministry of Interior, and its newly authorized committee developed the new regulation for ART services and also ART registry report forms which were adapted from ICMART. This new report will provide more useful data for strategic planning and development of ART services in Thailand.

\section{Conclusion}

Thailand has an excellent public health system but in recent years it has suffered from many ethical challenges regarding ART. The availability of assisted reproductive treatment is 
growing but remains limited and inaccessible for many of those who require treatment, with no public insurance and reimbursement available for infertility treatment in Thailand. The number of ART centers and ART cycles in Thailand increased between 2008 and 2014. Trends of the success rate did not increase during that period but the clinical pregnancy rate per transfer is better than the data reported previously between 2001 and 2007. After 2015, the new legislation restricts clinical practices for sex selection, limits eligibility for services, and bans all commercial egg donation or surrogacy or its facilitation. This research provides evidence that can be used to make public health recommendations and health care practice recommendations. It can also help individual providers and patients make informed decisions about infertility treatment and services.

Author contributions. KP contributed substantially to the conception and design of this study. Both authors contributed substantially to the acquisition of data. $\mathrm{CC}$ analyzed and interpreted the data and drafted the manuscript. KP contributed substantially to its critical revision. Both the authors approved the final version submitted for publication and take responsibility for the statements made in the published article.

Acknowledgment. The authors acknowledge the Royal Thai College of Obstetricians and Gynecologists for supporting the data of this research work.

Conflict of interest statement. The authors have completed and submitted the International Committee of Medical Journal Editors Uniform Disclosure Form for Potential Conflicts of Interest. None of the authors disclose any conflict of interest.

\section{References}

[1] Mascarenhas MN, Flaxman SR, Boerma T, Vanderpoel S, Stevens GA. National, regional, and global trends in infertility prevalence since 1990: a systematic analysis of 277 health surveys. PLoS Med. 2012; 9:e1001356.

[2] Okhovati M, Zare M, Zare F, Bazrafshan MS, Bazrafshan A. Trends in global assisted reproductive technologies research: a scientometrics study. Electron Physician. 2015; 7:1597-601.

[3] Kushnir VA, Barad DH, Albertini DF, Darmon SK, Gleicher N. Systematic review of worldwide trends in assisted reproductive technology 2004-2013. Reprod Biol Endocrinol. 2017; 15:6.

[4] Whittaker A. From mung ming to baby gammy: a local history of assisted reproduction in Thailand. Reprod Biomed Online. 2016; 2:71-8.

[5] Pruksananonda K, Suwajanakorn S, Boonkasemsanti W, Virutamasen P, Chinpilas V. Importance factors for the establishment of a successful program of intracytoplasmic sperm injection (ICSI). In: Gomel V, Leung PCK, editors. In vitro fertilization and assisted reproduction. Bologna:Monduzzi Editore; 1997, p. 537-40.

[6] Vutyavanich T, Piromlertamorn W, Ellis J; Reproductive Medicine Subcommittee, Royal Thai College of Obstetricians and Gynaecologists. Assisted reproductive technologies in Thailand: 2001-2007 results generated from the ART Registry, Royal Thai College of Obstetricians and Gynaecologists. J Obstet Gynaecol Res. 2011; 37:236-44.

[7] Pruksananonda K, Virutamasen P. Future concern for assisted reproductive technology in Thailand. Thai J Obstet Gynecol. 1997; 9:57-63.

[8] Whittaker A. International surrogacy as disruptive industry in Southeast Asia. New Brunswick, New Jersey: Rutgers University Press; 2018.

[9] Crooks VA, Turner L, Snyder J, Johnston R, Kingsbury P. Promoting medical tourism to India: messages, images, and the marketing of international patient travel. Soc Sci Med. 2011; 72:726-32.

[10] Whittaker A. Cross-border assisted reproduction care in Asia: implications for access, equity and regulations. Reprod Health Matters. 2011; 19:107-16.

[11] Virutamasen P, Pruksananonda K, Limpaphayom K, Chokevivat V, Kunaratanapruk $\mathrm{S}$. The regulation of assisted reproductive technology in Thailand. J Med Assoc Thai. 2001; 84:1490-4.

[12] Deonandan R. Recent trends in reproductive tourism and international surrogacy: ethical considerations and challenges for policy. Risk Manag Healthc Policy. 2015; 8:111-9.

[13] Sunderam S, Kissin DM, Crawford SB, Folger SG, Boulet SL, Warner L, Barfield WD. Assisted reproductive technology surveillanceUnited States, 2015. MMWR Surveill Summ. 2018; 67:1-28.

[14] Jones HW. Seven roads traveled well and seven to be traveled more. Fertil Steril. 2011; 95:853-6.

[15] Chambers GM, Paul RC, Harris K, Fitzgerald O, Boothroyd CV, Rombauts L, et al. Assisted reproductive technology in Australia and New Zealand: cumulative live birth rates as measures of success. Med J Aust. 2017; 207:114-8.

[16] European IVFMCftESoHR, Embryology, Calhaz-Jorge C, de Geyter C, Kupka MS, de Mouzon J, et al. Assisted reproductive technology in Europe, 2012: results generated from European registers by ESHRE. Hum Reprod. 2016; 31:1638-52.

[17] Mansour R, Ishihara O, Adamson GD, Dyer S, de Mouzon J, Nygren KG, et al. International Committee for Monitoring Assisted Reproductive Technologies world report: Assisted Reproductive Technology 2006. Hum Reprod. 2014; 29:1536-51.

[18] Zamora S, Clavero A, Gonzalvo MC, de Dios Luna Del Castillo J, Roldan-Nofuentes JA, Mozas J, et al. PGS-FISH in reproductive medicine and perspective directions for improvement: a systematic review. J Assist Reprod Genet. 2011; 28:747-57.

[19] Meldrum D. Society for assisted reproductive technology/centers for disease control and prevention in vitro fertilization registry 2.0. Fertil Steril. 2013; 100:1212-3.

[20] Boulet SL, Mehta A, Kissin DM, Warner L, Kawwass JF, Jamieson DJ. Trends in use of and reproductive outcomes associated with intracytoplasmic sperm injection. JAMA. 2015; 313:255-63.

[21] Calhaz-Jorge C, de Geyter C, Kupka MS, de Mouzon J, Erb K, Mocanu E, et al. Assisted reproductive technology in Europe, 2012:results generated from European registers by ESHRE. Hum Reprod 2016; 31 (8):1638-52.

[22] Ishihara O, Adamson GD, Dyer S, de Mouzon J, Nygren KG, Sullivan EA, et al. International committee for monitoring assisted reproductive technologies: world report on assisted reproductive technologies, 2007. Fertil Steril. 2015; 103:402-13. 